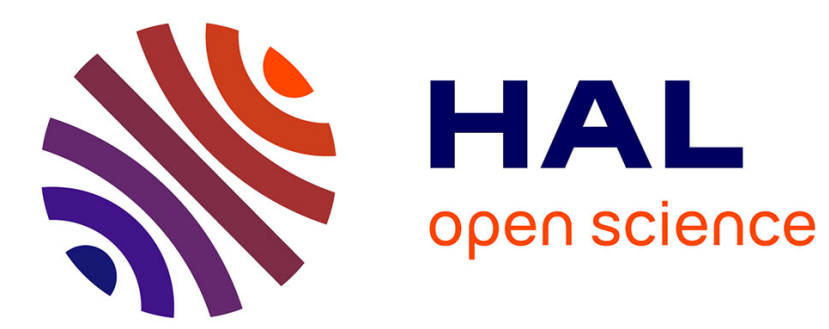

\title{
A stochastic model of the cell and tumor lifespans in radiotherapy
}

Thierry Bastogne, Roukaya Keinj, Pierre Vallois

\section{To cite this version:}

Thierry Bastogne, Roukaya Keinj, Pierre Vallois. A stochastic model of the cell and tumor lifespans in radiotherapy. 18th IFAC World Congress, IFAC WC'2011, Aug 2011, Milan, Italy. pp.CDROM, 10.3182/20110828-6-IT-1002.02073 . hal-00645164

\section{HAL Id: hal-00645164 \\ https://hal.science/hal-00645164}

Submitted on 27 Nov 2011

HAL is a multi-disciplinary open access archive for the deposit and dissemination of scientific research documents, whether they are published or not. The documents may come from teaching and research institutions in France or abroad, or from public or private research centers.
L'archive ouverte pluridisciplinaire HAL, est destinée au dépôt et à la diffusion de documents scientifiques de niveau recherche, publiés ou non, émanant des établissements d'enseignement et de recherche français ou étrangers, des laboratoires publics ou privés. 


\title{
A stochastic model of the cell and tumor lifespans in radiotherapy
}

\author{
T. Bastogne ${ }^{*}$ R. Keinj ${ }^{* *}$ P. Vallois ${ }^{* *}$ \\ * INRIA-BIGS \& Centre de Recherche en Automatique de Nancy \\ Nancy - Université, CNRS UMR 7039, \\ BP 70239, F-54506 Vandouvre-lès-Nancy Cedex, France, \\ Phone: (33) 383684473 - Fax: (33) 383684462 \\ ** INRIA-BIGS \& Institut de Mathématiques Elie Cartan, \\ Nancy-Université, CNRS UMR 7502, \\ BP 70239, F-54506 Vandouvre-lès-Nancy Cedex, France
}

\begin{abstract}
Classical models used in radiotherapy typically assume that after treatment all surviving cells have a constant and homogeneous sensitivity during the treatment period. In Keinj et al. (2010), a multinomial model based on a discrete-time Markov chain able to take into account both cell repair and cell damage heterogeneity due to radiations has been proposed. In this paper, we introduce the notion of lifespan for a single cell, a tumor and a normal tissue. We also determine the cumulative distribution functions of these random variables. These results lead to original formulations of the tumor control probability (TCP) and the normal tissue complication probability (NTCP). Finally, we propose a new characteristic: the Efficiency-Complication Trade-off diagram which provides to the radiotherapeutist all the information needed to choose the most appropriate treatment plan, i.e. the suited number of dose fractions to be applied to control the tumor while preserving the adjacent normal tissue. *
\end{abstract}

* Proc of the 18th IFAC World Congress. Invited session 'Modeling and Identification in Systems Biology: Advances and Challenges', Milano, Italy, Aug. 28 - Sep. 2, 2011

Keywords: dynamic models, stochastic models, multinomial distribution, cell lifespan, target theory, cancer

\section{INTRODUCTION}

Cancer is a disease that affects millions of people worldwide. One of the common therapies used to treat cancer is external beam radiotherapy. In this treatment, ionizing radiations are used to eliminate tumors. The ionizations induced by radiations cause a variety of possible lesions in cells, Curtis (1986). The most harmful damages are the lesions which affect the DNA structure, Feinendegen et al. (2008). Mathematical modelling may help to quantify the effects of the radioactive treatments on cell populations. It can be used to predict tumor growth and cancer spread, but also allows to determine the effectiveness of a specific treatment. Two probabilities are generally involved in the design of a treatment plan in radiotherapy : (i) the tumor control probability (TCP) and (ii) the normal tissue complication probability (NTCP). The TCP is the probability that all cancer cells are dead in the irradiated region, see Zaider and Minerbo (2000); Dawson and Hillen (2006). The NTCP is another probability that measures the sensitivity of normal tissue to radiations. The optimum choice of radiation dose delivery technique in the treatment of a given tumor is those that maximizes the TCP while keeping the NTCP lower than a given tolerance threshold. A classical method to obtain TCP, and probably the most used one, is to evaluate the probability that the surviving cell number, in the tumor, equals to zero. The expression of TCP and NTCP requires stochastic models and we are interested here in the simplest class of models used in radiotherapy: the hit models associated with the target theory. Note that other model structures, e.g. survival curves in Fowler
(1989), stochastic cell population-dynamic models in Sachs et al. (2001)or the cell-cycle models, in Kirkby et al. (2002), are also used to determine TCP and NTCP.

The target theory and hit-modeling paradigm were introduced in the 1920s when biologists were beginning to apply quantum physics to biology, see Dessauer (1922); Blau and Altenburger (1922). The modeling of radiation effects on living cells was initiated theoretically and experimentally by Lea and Catcheside (1942), and continued by Atwood and Norman (1949), and Pollard et al. (1955). In this theoretical setting, it is assumed that specific regions of the DNA called targets must be all inactivated to kill the cell. Each target is deactivated when it is hit by a number of radiation particules. Several classes of hit models classified by the number of targets and the number of hits were proposed, see Satow and Kawai (2006); Chapman (2007); Ditlov (2009).

Unfortunately two main aspects of tumor growth are often missing in target and hit models: (i) the target reparation between two consecutive dose fractions and (ii) the heterogeneity of damages induced by radiations in the cancer cell population after each dose fraction. In Keinj et al. (2010), an extension of hit models is proposed through a multinomial model that takes into account the different states of a surviving cell corresponding to the degree of DNA damage. Moreover, the proposed model is able to describe both target reparation between two consecutive dose fractions of the radiation schedule and the heterogeneity of damages induced by radiations in the cancer cell population after each dose fraction. In this multinomial model, a cell is 
supposed to contain $m$ targets which must be all deactivated to produce cell death. The cell population is then split up into $m+$ 1 categories. Like the majority of models used to measure the cell mortality, the multinomial model examines the number of surviving cells in a population. In this paper, we are interested in developing this model for the following objectives:

- to introduce the lifespan of a single cell, a tumor and a normal tissue and to study the randomness of these lifespans by determining their mean values, variances and their cumulative distribution functions;

- to use the previous probabilistic features to provide original formulations of TCP and NTCP. A concrete application of these results is to estimate the suitable dose to both control the tumor and limit the damage in healthy tissues.

In Section 2 we give a reminder on the tumor growth multinomial model. The modeling of the cancer cell lifespan $T$ is developed in Section 3. We then introduce the tumor and normal tissue lifespans $L_{n_{0}}$ and $L_{\bar{n}, \bar{n}_{0}}$ in Section 4 and we give new expressions of TCP and NTCP. Before concluding, we also propose a new diagram that summarizes all the information needed to choose the appropriate treatment schedule.

\section{MULTINOMIAL MODEL}

In Keinj et al. (2010), we have proposed a multinomial model of tumor growth relying on the target and hit modeling paradigm. This model is based on the following assumptions :

- a cell has $m$ targets;

- each target may be made inactive by a single hit caused by a radiation particule;

- the cell death happens when the $m$ targets are deactivated;

- between two consecutive dose fractions, if the cell is still alive then a target repair mechanism occurs;

- fractionated radiation doses are delivered at times $k=$ $1,2,3, \cdots, k$ is the number of treatment days.

\subsection{Heterogeneity of cell states after radiations}

We have $m+1$ possible states for a cell:

- state $i$, the cell has $i$ inactive targets, $i \in\{0,1, \ldots, m-1\}$, they are the $m$ states of a surviving cell;

- state $m$, the cell having $m$ inactive targets is a dead cell.

Figure 1 shows the case of a 3-target cell and the corresponding cell states. Let $Z_{k}$ the random variable denoting the state of
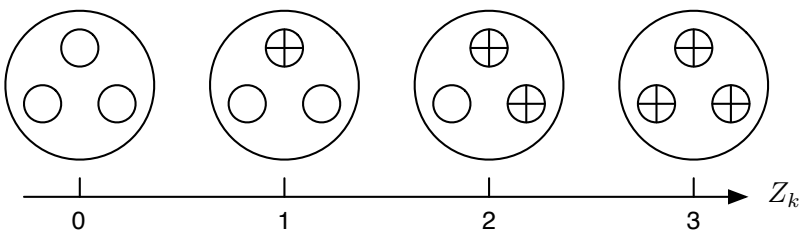

Fig. 1. 3-target cell, active and inactivated (crossed circles) targets and damage state $Z_{k}$ at time $k$

the cell at time $k, Z_{k}=i \in\{0,1, \cdots, m\}$ is the number of deactivated targets. We suppose that $\left(Z_{k}\right)$ is a discrete-time Markov chain, i.e. the cell state at time $k+1$ only depends on the current state at time $k$. Let $\Pi$ be the corresponding transition matrix. Moreover, $m$ is an absorbing state, because when the cell is died, it remains in state $m$ with a probability equal to 1 . We model the dynamic of the Markov chain $\left(Z_{k}\right)_{k \in \mathbf{N}}$ by taking firstly the effects of dose fractions, and secondly repair mechanisms into account as follows

$$
\Pi=\mathbf{P R} \text {, }
$$

where $\mathbf{P}$ models the effects of dose fractions, and $\mathbf{R}$ describes repair mechanisms. In this paragraph, the first row and first column of a matrix will be noted by the index value 0 .

\subsection{Treatment effect modelling}

Let $\mathbf{P}(i, j)$ the probability to deactivate $j$ targets at time $k+1$ when $i$ targets are disabled at time $k$. We assume that

- an active target becomes inactive with probability $q$;

- a deactivated target remains inactive with probability 1 ;

- the disabling of targets in the cell are independent events.

Therefore, if $i$ targets are inactive then after applying a new fraction dose we obtain $j$ inactive targets with probability

$$
\mathbf{P}(i, j)= \begin{cases}\left(\begin{array}{c}
m-i \\
j-i
\end{array}\right) q^{j-i}(1-q)^{m-j} & i \leq j \\
0 & j<i .\end{cases}
$$

\subsection{Cellular reparation modelling}

We introduce now repair mechanisms of deactivated targets which occur between two consecutive dose fractions. We suppose that

- a deactivated target in an alive cell can be repaired with probability $r$;

- an active target remains active with probability 1 ;

- the behaviors of the targets are independent.

Therefore, a cell in state $i<m$ at time $k$ may switch at time $k+1$ to the state $j \leq i$ if $i-j$ targets among the $i$ inactive targets are repaired and with probability

$$
\mathbf{R}(i, j)= \begin{cases}\left(\begin{array}{c}
i \\
j
\end{array}\right) r^{i-j}(1-r)^{j} & j \leq i<m \\
0 & i<j .\end{cases}
$$

When $i=m$, i.e. the cell is dead, the only possible case is $j=m$, then $\mathbf{R}(m, m)=1$.

\subsection{Probability distribution of $Z_{k}$}

Set $v_{k}=\left(v_{k}^{0}, \ldots, v_{k}^{m}\right)$ the probability distribution vector of $Z_{k}$ with $v_{k}^{i}=\operatorname{Pr}\left(Z_{k}=i\right)$. From the transition matrix $\Pi$ and the initial probability distribution $v_{0}$ of $Z_{0}$, we can determine $v_{k}$, for all $k \in \mathbf{N}^{*}$, using the Markov chain property,

$$
v_{k}=v_{0} \Pi^{k} \text {. }
$$

If we assume that all cells are initially in state $i_{0}\left(i_{0}\right.$ inactive targets $)$, then $\operatorname{Pr}\left(Z_{0}=i_{0}\right)=1$ and $v_{0}=\left(v_{0}^{0}, \ldots, v_{0}^{i_{0}}, \ldots, v_{0}^{m}\right)=$ $(0, \ldots, 1, \ldots, 0)$. Therefore, we obtain

$$
v_{k}^{i}=\Pi^{k}\left(i_{0}, i\right) \quad i \in\{0, \ldots, m\} .
$$

\subsection{Multinomial model of the tumor}

Consider a group of $n_{0}$ cells that behave independently and with the same dynamic. We suppose that each cell has $m$ targets and admits alternative damage and repair phases as explained at the begining of this section. For $i \in\{0, \ldots, m\}$, let the random 
variable $X_{k}(i)$ denote the number of cells in state $i$, among the $n_{0}$ initial cells, at time $k$ :

$$
X_{k}(i)=\sum_{l=1}^{n_{0}} 1_{\left\{Z_{k}^{(l)}=i\right\}}, \quad i \in\{0,1, \cdots, m\},
$$

where $Z_{k}^{(l)}$ is the number of inactive target(s) in the $l^{\text {th }}$ cell. From our assumptions, $Z_{k}^{(1)}, \ldots, Z_{k}^{\left(n_{0}\right)}$ are i.i.d. and distributed as $Z_{k}$. Consequently, the random vector $\left(X_{k}(0), \cdots, X_{k}(m)\right)$ follows a multinomial distribution with parameters $n_{0}$ and $v_{k}=$ $\left(v_{k}^{0}, \ldots, v_{k}^{m}\right)$ given in (5). Therefore,

$P\left(X_{k}(0)=a_{0}, \cdots, X_{k}(m)=a_{m}\right)=\frac{n_{0} !}{a_{0} ! \cdots a_{m} !}\left(v_{k}^{0}\right)^{a_{1}} \cdots \cdot\left(v_{k}^{m}\right)^{a_{m}}$

where $a_{0}, \cdots, a_{m} \in\left\{1, \cdots, n_{0}\right\}$ and $a_{0}+\cdots+a_{m}=n_{0}$. The number $X_{k}(i)$ of cells in state $i$ at time $k$ follows the binomial distribution with parameters $n_{0}$ and $v_{k}^{i}=\Pi^{k}\left(i_{0}, i\right)$,

$$
X_{k}(i) \sim \mathscr{B}\left(n_{0}, \Pi^{k}\left(i_{0}, i\right)\right) .
$$

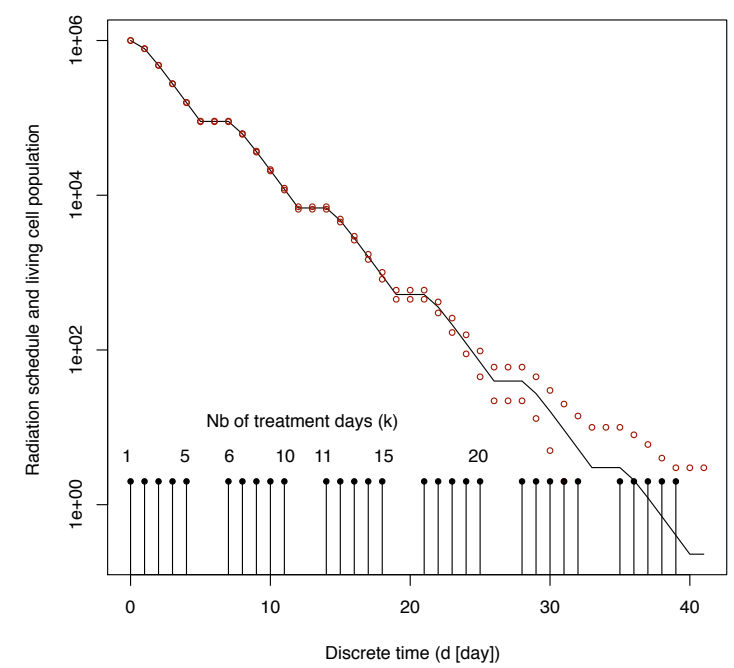

Fig. 2. Fractionated radiation schedules (black bullets) with 5 dose fractions per week, and the mean size of the living cell population: $n_{0}-E\left(X_{k}(m)\right)$ where $k$ denotes the number of dose fractions in the treatment schedule and $d$ is the discrete time based on a daily sampling rate. Red circles show the $99.9 \%$ confidence interval on the population size.

\section{LIFESPAN $T$ OF A CANCER CELL}

\subsection{Cell lifespan: definition and properties}

In this section, we develop an original approach based on the lifespan of a cancer cell which has received a sequence of successive dose fractions of radiation. The cell lifespan is the first (random) instant of treatment time when the cell is dead. We would like to emphasize this new viewpoint of cell mortality modeling. Indeed, in the usual hit models and in the previous multinomial model, $k$ is fixed and we only consider random variables at time $k, e . g$. the number $Z_{k}$ of deactivated targets. In the multinomial setting it has been proved that the distribution of $Z_{k}$ is given by $\Pi^{k}$.
Now, we consider a random variable $T$ denoting the lifespan of a single cell composed of $m$ targets. In other terms, $T$ is the first instant $k$ when the cell is in state $m$ (death state) :

$$
T=\inf \left\{i \geq 1 ; Z_{i}=m\right\} \text {. }
$$

The knowledge of $T$ allows to recover information at time $k$ for instance $T \leq k$ is equivalent to $Z_{k}=m$. Moreover $T$ reveals the dynamical aspect of the cell lifespan, so $\{T=k\}=\left\{Z_{1} \neq\right.$ $\left.m, \cdots, Z_{k-1} \neq m, Z_{k}=m\right\}$ and therefore involves the sequence $Z_{1}, \cdots, Z_{k}$ instead of $Z_{k}$ only. $T$ gives naturally rise to Monte Carlo simulations.

The counterpart is that we have to deal with a random element. In Proposition 3.1 below, we calculate its mean time and the associated variance. The second parameter permits to control the variability of $T$ around its mean value. This rough information can be completed by the cumulative distribution which highlights how $T$ fluctuates.

Proposition 3.1. If the cell is initially in state $Z_{0}=i_{0} \in$ $\{0, \ldots, m-1\}$, the random variable $T$ of the cancer cell lifespan verifies the following properties :

(1) The cumulative distribution function $F$ of $T$ is related to the transition matrix $\Pi$ :

$$
F(k)=P(T \leq k)=\Pi^{k}\left(i_{0}, m\right) .
$$

(2) For all $n \in \mathbb{N}^{*}$, the expected value $E\left(T^{n}\right)$ is finite and

$$
E(T)=\sum_{k \geq 1} k\left(\Pi^{k}\left(i_{0}, m\right)-\Pi^{k-1}\left(i_{0}, m\right)\right) .
$$

(3) The variance $V(T)$ is finite and given by

$$
V(T)=\sum_{k \geq 1} k^{2}\left(\Pi^{k}\left(i_{0}, m\right)-\Pi^{k-1}\left(i_{0}, m\right)\right)-(E(T))^{2} .
$$

Proof 1. According to the definition of $T$ (cf (9)) and the crucial fact that $m$ is an absorbant state we have

$$
P(T \leq k)=P\left(Z_{k}=m \mid Z_{0}=i_{0}\right) \quad \forall k \in \mathbb{N}^{*} .
$$

According to (5), we obtain

$$
F(k)=P(T \leq k)=\Pi^{k}\left(i_{0}, m\right) .
$$

2. Set

$$
\begin{aligned}
\lambda_{1} & =\max \{1-\Pi(i, m), 0 \leq i \leq m-1\} \\
& =\max \left\{\sum_{j \leq m-1} \Pi(i, j), 0 \leq i \leq m-1\right\} \\
\lambda_{2} & =\max \{\Pi(i, m), 0 \leq i \leq m-1\} \\
\lambda & =\max \left(\lambda_{1}, \lambda_{2}\right) .
\end{aligned}
$$

Note that, $\lambda_{1}<1$ and $\lambda_{2}<1$ then $\left.\lambda \in\right] 0,1[$. We prove that

$$
P(T=k) \leq \lambda_{2} \lambda_{1}^{k-1} \leq \lambda^{k}
$$

only when $k=3$. The general proof being similar:

$$
\begin{aligned}
P(T=3) & =P\left(Z_{1} \neq m, Z_{2} \neq m, Z_{3}=m\right) \\
& =\sum_{i_{1}, i_{2} \leq m-1} \Pi\left(i_{0}, i_{1}\right) \Pi\left(i_{1}, i_{2}\right) \Pi\left(i_{2}, m\right) \\
& \leq \lambda_{2}\left(\sum_{i_{1} \leq m-1}\left(\sum_{i_{2} \leq m-1} \Pi\left(i_{1}, i_{2}\right)\right) \Pi\left(i_{0}, i_{1}\right)\right) \\
& \leq \lambda_{2} \lambda_{1}\left(\sum_{i_{1} \leq m-1} \Pi\left(i_{0}, i_{1}\right)\right) \leq \lambda_{2} \lambda_{1}^{2} \leq \lambda^{3} .
\end{aligned}
$$

As $\lambda \in] 0,1\left[\right.$, we deduce that the mean value $E\left(T^{n}\right)<\infty, n \geq 1$ : 


$$
\begin{aligned}
E\left(T^{n}\right) & =\sum_{k \geq 1} k^{n} P(T=k) \\
& \leq \sum_{k \geq 1} k^{n} \lambda^{k}<\infty
\end{aligned}
$$

For $n=1, E\left(T^{n}\right)=E(T)<\infty$. Now, we express $E(T)$ in terms of the cumulative distribution function $F$. Thus,

$$
\begin{aligned}
E(T) & =\sum_{k \geq 1} k(P(T<k+1)-P(T<k)) \\
& =\sum_{k \geq 1} k(F(k)-F(k-1)) . \\
& =\sum_{k \geq 1} k\left(\Pi^{k}\left(i_{0}, m\right)-\Pi^{k-1}\left(i_{0}, m\right)\right) .
\end{aligned}
$$

3. For $n=2, E\left(T^{2}\right)<\infty$ and the variance of $T$ is finite as it given by:

$$
\begin{aligned}
V(T) & =E\left(T^{2}\right)-(E(T))^{2} \\
& =\sum_{k \geq 1} k^{2}(P(T<k+1)-P(T<k))-(E(T))^{2} \\
& =\sum_{k \geq 1} k^{2}\left(\Pi^{k}\left(i_{0}, m\right)-\Pi^{k-1}\left(i_{0}, m\right)\right)-(E(T))^{2} .
\end{aligned}
$$

\subsection{Confidence interval of $T$}

We are interested in a most probable interval $I$ i.e. $I$ such that

$$
P(T \in I) \geq 1-\alpha,
$$

where $1-\alpha$ is the confidence level. We restrict ourselves to interval $I$ of the type $\left.I=] E(T)-\varepsilon_{1}, E(T)+\varepsilon_{2}\right]$. The positive real numbers $\varepsilon_{1}$ and $\varepsilon_{2}$ are determined by the conditions :

$$
P\left(T \leq E(T)-\varepsilon_{1}\right) \leq \beta_{1} \quad \text { and } \quad P\left(T>E(T)+\varepsilon_{2}\right) \leq \beta_{2},
$$

where $\beta_{1}+\beta_{2}=\alpha$. For instance, $\alpha=0.05, \beta_{1}=\beta_{2}=\frac{\alpha}{2}=$ 0.025 .

The confidence interval $I$ represents values of $T$ for which the difference between $T$ and $E(T)$ is not statistically significant at the $\alpha$ level. $F$ being the cumulative distribution function of $T$, it is clear that (20) is equivalent to

$$
F\left(E(T)-\varepsilon_{1}\right) \leq \beta_{1} \quad \text { and } \quad F\left(E(T)+\varepsilon_{2}\right) \geq 1-\beta_{2} .
$$

\subsection{Numerical analysis}

We present here a set of numerical experiments to show the influence of the parameters $q, r$ and the initial state $Z_{0}=i_{0}$, on the interval $I$ in the case $\beta_{1}=\beta_{2}=\frac{\alpha}{2}=0.025$. We fix two parameters and we vary the third one. We implemented the cumulative distribution function $F$ on Matlab with $m=3$. Table 1, figures 3 and 4 present all the numerical results.

We can observe and quantify numerically the monotonicity of the confidence interval $I$, specially when the efficiency of the treatment or the repair mechanisms are changed. It also appears that the interval $I$ is smaller when the initial state of the cell is fragile and also when the treatment is more efficient. Conversely, the interval $I$ becomes larger when the cellular repair mechanisms are stronger.

\section{ORIGINAL FORMULATIONS OF TCP AND NTCP}

The TCP is the probability that all cancer cells are dead in the irradiated region and the NTCP measures the sensitivity

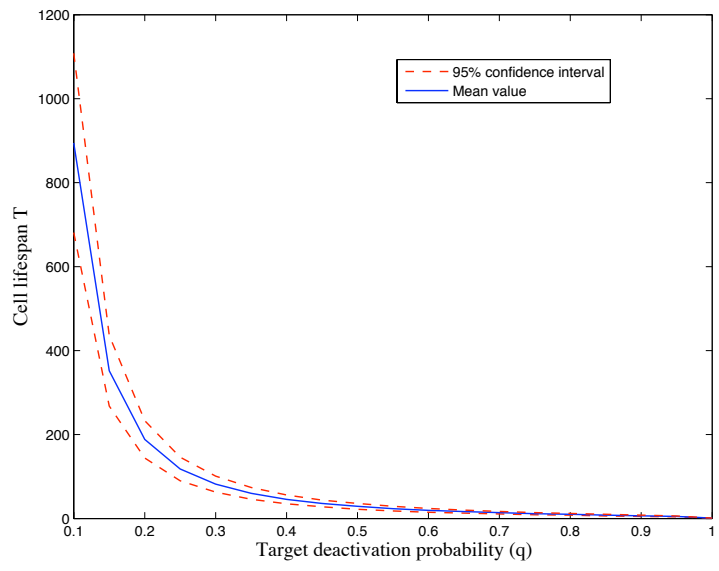

Fig. 3. Sensitivity of the cell lifespan $T$ to the target deactivation probability $q$

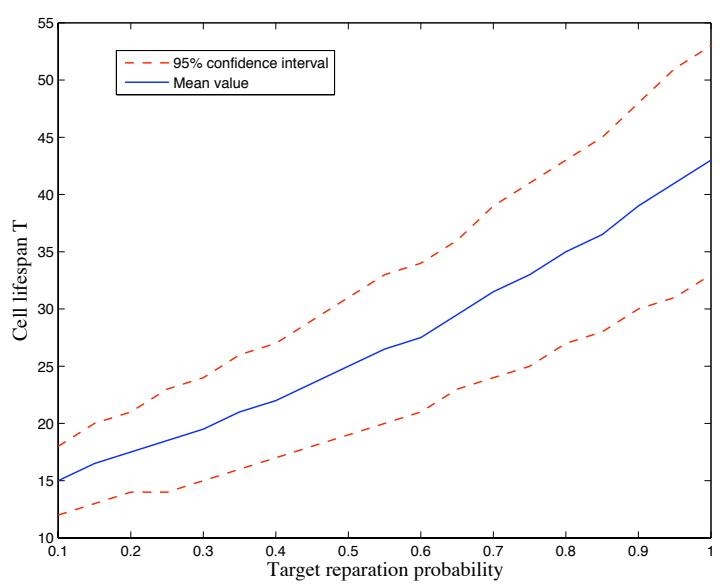

Fig. 4. Sensitivity of the cell lifespan $T$ to the target reparation probability $r$

\begin{tabular}{|c|c|c|c|}
\hline Fixed parameters & Varied parameter & $E(T)$ & $I$ \\
\hline$q=0.4$ & $Z_{0}=0$ & 5.76 & {$[1,17]$} \\
\cline { 2 - 4 }$r=0.3$ & $Z_{0}=2$ & 3.63 & {$[1,14]$} \\
\hline$r=0.3$ & $q=0.6$ & 2.90 & {$[1,7]$} \\
\cline { 2 - 4 }$Z_{0}=0$ & $q=0.8$ & 1.70 & {$[1,3]$} \\
\hline$q=0.6$ & $r=0.2$ & 2.75 & {$[1,6]$} \\
\cline { 2 - 4 }$Z_{0}=0$ & $r=0.6$ & 3.47 & {$[1,10]$} \\
\hline
\end{tabular}

Table 1. Influence of the multinomial parameters $q, r$ and $i_{0}$ on the confidence interval $I$ of $T$ with $m=3, n_{0}=10^{4}$ and $\beta_{1}=\beta_{2}=0.025$.

of the adjacent normal tissue for a given radiation treatment schedule. These probabilities are simultaneously used to optimize the quality of a treatment planning. The treatment goal is to maximizes the TCP and to keep the NTCP lower than a given tolerance threshold. In this section, we give an original approach based on the cell lifespan to choose the optimal dose fraction number for a treatment plan and we give new expressions of TCP and NTCP.

\subsection{Tumor Control Probability}

The tumor control probability at time $k$ is the probability to destroy the tumor at this time. If $L_{n_{0}}$ is the lifespan of the tumor, then $T C P_{k}$ is the probability that $L_{n_{0}}$ is lower than $k$,

$$
T C P_{k}=P\left(L_{n_{0}} \leq k\right) \text {. }
$$




\begin{tabular}{|c|c|c|}
\hline$n_{0}$ & $E\left(L_{n_{0}}\right)$ & $J$ \\
\hline \hline$n_{0}=10^{3}$ & 12.9 & {$[10 ; 18]$} \\
\hline$n_{0}=10^{6}$ & 23.6 & {$[21 ; 28]$} \\
\hline$n_{0}=10^{9}$ & 34.2 & {$[31 ; 39]$} \\
\hline$n_{0}=10^{12}$ & 44.9 & {$[42 ; 50]$} \\
\hline
\end{tabular}

Table 2. Influence of the initial cancer cell number $n_{0}$ on the expected value and confidence interval $J$ of $L_{n_{0}}$.

In order to find an explicit expression of $T C P_{k}$, we express $L_{n_{0}}$ in terms of the single cell lifespans.

Lifespan $L_{n_{0}}$ of the tumor. We consider a tumor initially composed of $n_{0}$ living cells. For each single cell $j$ we associate the corresponding lifespan:

$$
T^{(j)}=\inf \left\{i \geq 1 ; Z_{i}^{(j)}=m\right\} .
$$

$L_{n_{0}}$ corresponds to the time when the whole tumor is eliminated, i.e. all the cells are killed. $L_{n_{0}}$ can then be expressed with respect to the variables $\left\{T^{(j)}\right\}_{j \in\left\{1, \ldots, n_{0}\right\}}$ as

$$
L_{n_{0}}=\max \left\{T^{(1)}, \ldots, T^{\left(n_{0}\right)}\right\} \text {. }
$$

Consequently,

$$
P\left(L_{n_{0}} \leq k\right)=P\left(T^{(1)} \leq k, \ldots, T^{\left(n_{0}\right)} \leq k\right) .
$$

Under the assumption that all cells behave independently and with the same dynamic, $T^{(1)}, \ldots, T^{\left(n_{0}\right)}$ are then i.i.d. random variables and have the same distribution as $T(\mathrm{cf}(11))$. Therefore, we obtain

$$
\begin{aligned}
P\left(L_{n_{0}} \leq k\right) & =P\left(T^{(1)} \leq k\right) \times \ldots \times P\left(T^{\left(n_{0}\right)} \leq k\right) \\
& =(P(T \leq k))^{n_{0}} .
\end{aligned}
$$

According to Proposition 3.1, equation (17) and the assumption that all cells are initially in state $i_{0}$, we have

$$
T C P_{k}=\left(\Pi^{k}\left(i_{0}, m\right)\right)^{n_{0}}
$$

Confidence interval of $L_{n_{0}}$. Let $J$ the confidence interval of $L_{n_{0}}$ of the type $\left.\left.J=\right] E\left(L_{n_{0}}\right)-\kappa_{1}, E\left(L_{n_{0}}\right)+\kappa_{2}\right]$ and we determine the positive real numbers $\kappa_{1}$ and $\kappa_{2}$ by the conditions :

$$
P\left(L_{n_{0}} \leq E\left(L_{n_{0}}\right)-\kappa_{1}\right) \leq 0.025
$$

and

$$
P\left(L_{n_{0}}>E\left(L_{n_{0}}\right)+\kappa_{2}\right) \leq 0.025 .
$$

These conditions are equivalent to

$$
G\left(E\left(L_{n_{0}}\right)-\kappa_{1}\right) \leq 0.025 \quad \text { and } \quad G\left(E\left(L_{n_{0}}\right)+\kappa_{2}\right) \geq 0.975 \text {, }
$$

where $G(\cdot)$ denotes the cumulative distribution function of $L_{n_{0}}$, i.e. $G(k):=P\left(L_{n_{0}} \leq k\right)$.

\section{Numerical analysis}

We implemented the cumulative distribution function $G$ and $E\left(L_{n_{0}}\right)$ in Matlab. Numerical results are given in table 4.1.2. The mean lifespan of the tumor increases with $n_{0}$ while the width of the confidence interval remains almost constant.

\subsection{Normal Tissue Complication Probability}

Normal Tissue Complication Probability (NTCP) is the probability that a complication appears in the adjacent normal tissue. A complication occurs when the number of dead cells in the normal tissue is larger than a given threshold number $\bar{n}$.
We suppose that the normal cells behave similarly than the cancer cells, the difference coming from different values of the model parameters. A normal cell contains $\bar{m}$ targets, each of them is deactivated with a probability $\bar{q}$ and it is repaired with a probability $\bar{r}$. For simplicity, we use the same notation, as for cancer cells, to denote the probability $\bar{\Pi}^{k}\left(\bar{i}_{0}, \bar{m}\right)$ that a normal cell is in state $\bar{m}$ at time $k$ (dead cell).

Lifespan $\bar{T}$ of a normal cell. We consider a single normal cell that contains $\bar{m}$ targets. Let $\bar{Z}_{k}$ be the random variable denoting the state of the normal cell at time $k$ and $\bar{T}$ its lifespan. Similary to the case of a cancer cell, $\bar{T}$ is defined as

$$
\bar{T}=\inf \left\{i \geq 1 ; \bar{Z}_{i}=\bar{m}\right\},
$$

and the corresponding cumulative distribution function is

$$
\bar{F}(k)=P(\bar{T} \leq k)=\bar{\Pi}^{k}\left(\bar{i}_{0}, \bar{m}\right) .
$$

Lifespan $L_{\bar{n}, \bar{n}_{0}}$ of the normal tissue. Consider now a normal tissue containing initially $\bar{n}_{0}$ cells and we define $L_{\bar{n}, \bar{n}_{0}}$ the random variable denoting the lifespan of the entire normal tissue. For each cell $j$ we associate the corresponding lifespan,

$$
\bar{T}^{(j)}=\inf \left\{i \geq 1 ; \bar{Z}_{i}^{(j)}=\bar{m}\right\} .
$$

Let us rearrange these random variables into a nondecreasing sequence

$$
\bar{T}^{\left(1: \bar{n}_{0}\right)} \leq \bar{T}^{\left(2: \bar{n}_{0}\right)} \leq \ldots \leq \bar{T}^{\left(\bar{n}_{0}: \bar{n}_{0}\right)}
$$

where $\bar{T}^{\left(l: \bar{n}_{0}\right)}$ denotes the lifespan of $l$ normal cells among $\bar{n}_{0}$. This sequence is called the order statistics of $\bar{T}^{(1)}, \bar{T}^{(2)}, \ldots, \bar{T}^{\left(\bar{n}_{0}\right)}$. $\bar{T}^{\left(l: \bar{n}_{0}\right)}$ is called the $l^{\text {th }}$ order statistic.

Proposition 4.1. The normal tissue lifespan $L_{\bar{n}, \bar{n}_{0}}$ is the $\bar{n}^{t h}$ order statistic and the NTCP at time $k$ is given by

$$
N T C P_{k}=P\left(L_{\bar{n}, \bar{n}_{0}} \leq k\right)=\sum_{i=\bar{n}}^{\bar{n}_{0}}\left(\begin{array}{c}
\bar{n}_{0} \\
i
\end{array}\right) \bar{F}^{i}(k)(1-\bar{F}(k))^{\bar{n}_{0}-i} \text {. }
$$

Proof Firstly, we determine the distribution of $\bar{T}^{\left(\bar{n}: \bar{n}_{0}\right)}$. As a matter of fact, if we put

$$
A_{j}(k)=\left\{\bar{T}^{(j)} \leq k\right\}
$$

then

$$
\left\{\bar{T}^{\left(\bar{n}: \bar{n}_{0}\right)} \leq k\right\}=\left\{\sum_{j=1}^{\bar{n}_{0}} 1_{A_{j}(k)} \geq \bar{n}\right\} .
$$

As we have $P\left(A_{j}(k)\right)=\bar{F}(k)$ and the events $A_{1}(k), \cdots, A_{n_{0}}(k)$ are independent then $\sum_{j=1}^{\bar{n}_{0}} 1_{A_{j}(k)} \sim \mathscr{B}\left(\bar{n}_{0}, \bar{F}(k)\right)$ :

$$
P\left(\bar{T}^{\left(\bar{n}: \bar{n}_{0}\right)} \leq k\right)=\sum_{i=\bar{n}}^{\bar{n}_{0}}\left(\begin{array}{c}
\bar{n}_{0} \\
i
\end{array}\right)(\bar{F}(k))^{i}(1-\bar{F}(k))^{\bar{n}_{0}-i} .
$$

Finally, it is clear that NTCP at time $k$ is given by

$$
N T C P_{k}=P\left(L_{\bar{n}, \bar{n}_{0}} \leq k\right) \text {. }
$$

\subsection{Determination of a suited number of dose fractions}

The main task in radiotherapy is to choose the treatment schedule that permits to eliminate the tumor without causing significant damage on the adjacent normal tissue. This question may be reformulated as the choice of the appropriate treatment duration. To help the radiotherapeutist to answer this question, we propose a new diagram, entitled ECT (Efficiency Complication Trade-off) which is inspired from the ROC (Receiver Operating Characteristic) / DET (Detection Error Trade-off) curves used in biometrics. The ECT diagram allows to compute both NCTP (x-axis) and the treatment non-efficiency probability (1-TCP) 


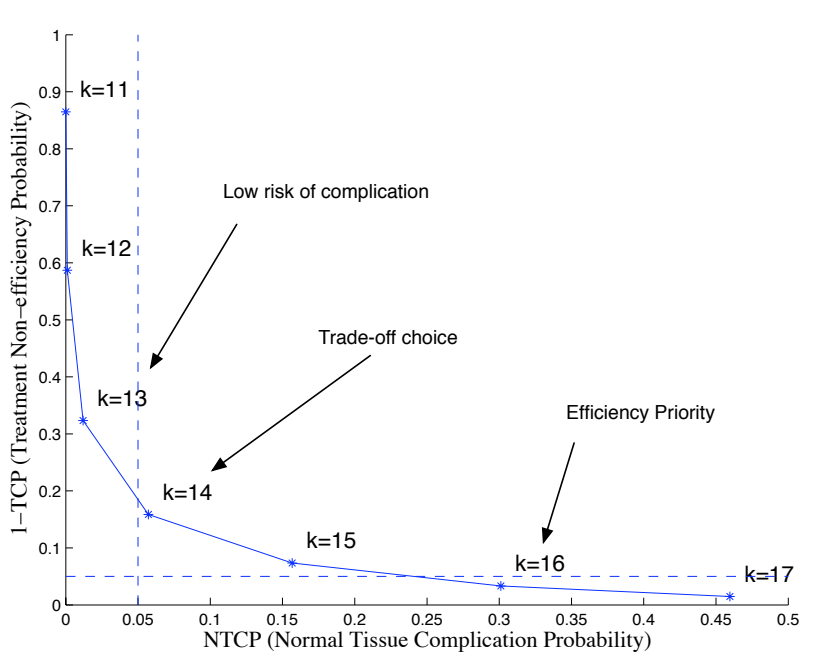

Fig. 5. ECT diagram (Efficiency Complication Trade-off)

on y-axis for different values of $k$ (number of treatment days). In this diagram, the ideal therapeutic goal is the origin point $\mathrm{O}$ $(0,0)$ since it corresponds to a $100 \%$ efficient treatment and a null risk of complication. Note that $k \rightarrow T C P_{k}$ and $k \rightarrow N T C P_{k}$ are increasing.

Figure 5 shows the ECT curve for $m=\bar{m}=3, q=0.7, \bar{q}=0.6$ $r=0.3, \bar{r}=0.2, n_{0}=10^{4}$, and $\bar{n}=\bar{n}_{0}=10^{3}$. We propose three strategies to select a suitable treatment duration:

- A first choice consists in limiting the probability of complication on normal tissues with a threshold fixed in this example at 0.05 (dotted vertical line). Beyond this level, the risk of complication is not acceptable. Subsequently, the most appropriate number of dose fractions to be applied is given by $k=13$ dose fractions with a treatment non-efficiency probability estimated at $1-T C P \approx 0.3$.

- A second choice is to give priority to the therapeutic efficiency by using an non-efficiency threshold fixed in this example to 0.05 (dotted horizontal line). This choice leads to choose at least $k=16$ days of treatment with a risk of complication estimated at NTCP $\approx 0.3$.

- A third possibility is a trade-off choice which consists in selecting the nearest point of the curve from the ideal point $\mathrm{O}(0,0)$. In this case, the choice leads to select $k=14$ days of treatment with a non-efficiency probability lower than 0.2 and a complication probability $N T C P \approx 0.05$.

\subsection{Limits}

The current multinomial model used to calculate the tumor lifespan, TCP and NTCP has two main limitations:

- it does not take by-standing effects into account in the cell mortality process;

- and numerical errors occur during Matlab computations when $n_{0}>10^{14}$. In other terms, this numerical problem currently limits the application of our model to avascular tumors $\left(<1 \mathrm{~mm}^{3}\right)$.

Nevertheless, studies are currently in progress to overcome these two computational limits.

\section{CONCLUSION}

We have proposed an original approach that expresses the probability distribution of the cancer and normal cells lifespans in terms of the treatment duration (or number of dose fractions) in radiotherapy. Our approach relies on a multinomial model, which is itself based on a discrete-time Markov chain. This model takes into account (i) the variety of cell responses according to their biological states and (ii) the repair mechanisms that occur between dose fractions. The main contribution is the introduction of two new response variables : the cancer cell and tumor lifespans, $T$ and $L_{n_{0}}$ respectively. A new expression of the TCP is proposed based on the cumulative distribution function of $L_{n_{0}}$. The $(1-\alpha)$ confidence intervals of $T$ and $L_{n_{0}}$ were also determined. We have also introduced the normal tissue lifespan $L_{\bar{n}, \bar{n}_{0}}$ and provided an original formulation of the NTCP in terms of its cumulative distribution function $G$ of $T$. Finally, we have proposed a new representation, called ECT (Efficiency-Complication Trade-off) which allows to optimize both efficiency and complication of the treatment. This characteristic could help the radiotherapeutist to choose the number of dose fractions suited to control the tumor while preserving the adjacent normal tissue.

\section{REFERENCES}

Atwood, K.C. and Norman, A. (1949). On the interpretation of multi-hit survival curves. Proc. Natl. Acad. Sci. USA, 35, 696-709.

Blau, M. and Altenburger, K. (1922). Uber einige Wirkungen von Strahlen. II. Z. Phys., 12(2), 315-329.

Chapman, J.D. (2007). Target theory revisited: Why physicists are essential for radiobiology research. Clinical Oncology, 19, S12.

Curtis, S.B. (1986). Lethal and potentially lethal lesions induced by radiation - a unified repair model. Radiat. Res, 106, 252-279.

Dawson, A. and Hillen, T. (2006). Derivation of the tumour control probability (tcp) from a cell cycle model. Computational and Mathematical Methods in Medicine, 7, 121-141.

Dessauer, F. (1922). Uber einige Wirkungen von Strahlen. I. Z. Phys., 12, 38-47.

Ditlov, V.A. (2009). Track theory and radiation effects. Radiation Measurements, 44, 1100-1104.

Feinendegen, L., Hahnfeldt, P., Schadt, E.E., Stumpf, M., and Voit, E.O. (2008). Systems biology and its potential role in radiobiology. Radiat Environ Biophys, 47, 5-23.

Fowler, J. (1989). The linear-quadratic formula and progress in fractionated radiotherapy. Br. J Radiol, 62, 679-694.

Keinj, R., Bastogne, T., and Vallois, P. (2010). A multinomial model of tumour growth treated by radiotherapy. In Proceedings of the 11th IFAC Symposium on Computer Applications in Biotechnology; Leuven, Belgium.

Kirkby, N.F., G, B.N., and Faraday, D.B.F. (2002). Mathematical modelling of the response of tumour cells to radiotherapy. Nuclear Instruments and Methods in Physics Research. Section B: Beam Interactions with Materials and Atoms, 188, 210-215.

Lea, D.E. and Catcheside, D.G. (1942). The mechanism of induction by radiation of chromosome aberrations in tradescantia. J. Genet., 44, 216-45.

Pollard, E.C., Guild, W.R., Hutchinson, F., and Setlow, R.B. (1955). The direct action of ionizing radiation on enzymes and antigen. Progress in Biophysics, 5, 72-108. 
Sachs, R.K., Hlatky, L.R., and Hahnfeldt, P. (2001). Simple ODE models of tumor growth and anti-angiogenic or radiation treatment. Mathematical and Computer Modelling, 33, 1297-1305.

Satow, T. and Kawai, H. (2006). Hit and target models for dna damage with indirect action. Computers and Mathematics with Applications, 51, 257-268.

Zaider, M. and Minerbo, G.N. (2000). Tumour control probability: a formulation applicable to any temporal protocol of dose delivery. Phys. Med. Biol., 45, 279-293. 